\title{
Molecular Docking and Clinical study of inhibition of \\ Phytochemical compounds of Nigella sativa, Matricaria \\ chamomilla and Origanum vulgare L on COVID_19 Mpro
}

Neda Shaghaghi, Sima Fereydooni, Naiemeh Molaei kordabad

Institute: Center for the growth of drug units, tbzmed

\begin{abstract}
Due to the high rate of infection reported in the new Coronavirus, in recent months, a lot of research has been done on the introduction of antiviral drugs. Recent studies have shown that inhibiting viral protease enzymes are highly effective in controlling infection caused by any type of virus. The aim of this study was to investigate the bioinformatics of inhibition of the new coronavirus protease enzyme by compounds in the essential oils of three medicinal plants. This is a descriptive-analytical study. For this bioinformatics analysis, the structure of compounds from PubChem database and the structure of COVID_19 protease enzyme were obtained from PDB database. Molecular docking was then performed by MVD software. The results showed that the site of interaction of the compounds in the protected area is enzymatic flap. Also, 15 patients voluntarily received steam therapy with the essential oils of these plants and their symptoms of lung infection improved. Therefore, it can be concluded that the studied compounds with strong interaction due to their natural origin and the possibility of less side effects, as well as their direct entry into the respiratory tract, these compounds are suitable for drug treatment for coronavirus infection.
\end{abstract}

Keyword : Coronavirus, Bioinformatic, Molecular docking, Plant Drug

\section{Introduction}

The Coronavirus infection is a disease associated with respiratory problems, which can lead to death from respiratory failure. About 210 countries and territories have been reported to be infected with the virus in the United States, China, South Korea, Italy, Spain, France, Russia, 
Turkey, Iran, etc. This article is dedicated to making an essential oil remedy for respiratory and inflamatory problems. Coronaviruses belong to the Coronaviridae family and appear just like spiked rings when observed through an electron microscope. The surface looks with various spikes, which are helpful to attack and bind living cells. These are the viruses causing the simple common cold disease to severe illnesses like Middle East Respiratory Syndrome (MERS-CoV), Severe Acute Respiratory Syndrome (SARS-CoV). The source of these viruses is some animals including bats. The word coronavirus is a derivative of the Latin corona, which means crown or halo, that states to the typical look indicative of a crown or a solar corona around the virions. These viruses are having a positive-sense single-stranded RNA genome (27 to 34 kilobases) and helical symmetry nucleocapsid (Sexton et al., 2016; S. Su et al., 2016). It should be noted that the outbreak of 2019 to 2020 in Wuhan, Hubei Province, China began in December 2019 when a new species of coronavirus was discovered on December 31, 2019. The World Health Organization (WHO) has named the virus 2019nCoV (Novel Coronavirus 2019, 2020), which was later renamed by the International Committee for the Classification of Viruses as Acute Respiratory Syndrome Coronavirus 2 (SARS-CoV-2). Named. Diseases caused by the virus are referred to as Coronavirus 2019 and COVID-19 for short (I. Ali \& Alharbi, 2020).

The most important use of essential oils is to make them fragrant. Essential oils also have medicinal, industrial and food uses. They are also used in cosmetics. Many essential oils have antiseptic properties. Among the effects of essential oils are the effect of improving blood flow, anti-inflammatory effect, for example, in chamomile,.Their disinfectant effect on cellular metabolism. Their bacteriostatic effect can be due to their effect on surface tension.The mechanism of antibacterial and antifungal effects of a number of essential oils in animal models has been studied, including three mechanisms: dissolution in the cytoplasmic membrane and the effect on the enzyme structure and destruction of microorganisms. Oxidative, anti-inflammatory and antispasmodic effect of essential oils. (Sharifi-Rad et al., 2017).

In the field of molecular modeling, docking is a method which predicts the preferred orientation of one molecule to a second when bound to each other to form a stable complex.To perform a docking screen, the first requirement is a structure of the protein of interest. Usually the structure has been determined using a biophysical technique such as $\mathrm{x}$ ray crystallography or NMR spectroscopy, but can also derive from homology modeling construction. This protein structure and a database of potential ligands serve as inputs to a 
docking program. A binding interaction between a small molecule ligand and an enzyme protein may result in activation or inhibition of the enzyme. If the protein is a receptor, ligand binding may result in agonism or antagonism. Docking is most commonly used in the field of drug design (Huang et al, 2006; Lengauer \& Rarey, 1996).

\section{Method}

This research was a descriptive-analytical study. In this study, the interaction of compounds as described in Table 2 was investigated. In order to obtain the 2 and 3 dimensional structure of the compounds, a PubChem database (https://pubchem.ncbi.nlm.nih.gov) was used. The PDB database (https://www.rcsb.org/) was used to obtain the complete structure of the protease enzyme.The structure mentioned in access number 6lu7 was received in the PDB database (Liu et al., 2020). Also, in this study, 15 patients volunteered to use this combination with the steam treatment method and once a day, they received a steam treatment with a specific percentage of the combination of plants mentioned in this study.

Table 1.Name and structure of the compounds studied

\begin{tabular}{|c|c|c|}
\hline Compound Name & Plant Sourse & \\
\hline \multirow{3}{*}{ Dithymoquinone } & Nigella sativa & \\
\hline \multirow{3}{*}{ Farnesene } & Matricaria chamomilla & \\
\hline
\end{tabular}




\begin{tabular}{|c|c|c|}
\hline Spathulenol & Matricaria chamomilla \\
\hline Chamazulene & Matricaria chamomilla & \\
\hline $\begin{array}{c}\text { En-yn- } \\
\text { dicycloether }\end{array}$ & Matricaria chamomilla & \\
\hline $\begin{array}{c}\text { Thymol } \\
\text { Luteolin }\end{array}$ & Matricaria chamomilla & \\
\hline & & \\
\hline
\end{tabular}




\begin{tabular}{|c|c|c|}
\hline Geranyl_acetate & Origanum vulgare L \\
\hline Borneol acetate & Origanum vulgare $L$ & \\
\hline beta- & Origanum vulgare $L$ & \\
\hline Germacren D & Origanum vulgare $L$ & \\
\hline beta-Bourbonene & Origanum vulgare $L$ & \\
\hline
\end{tabular}




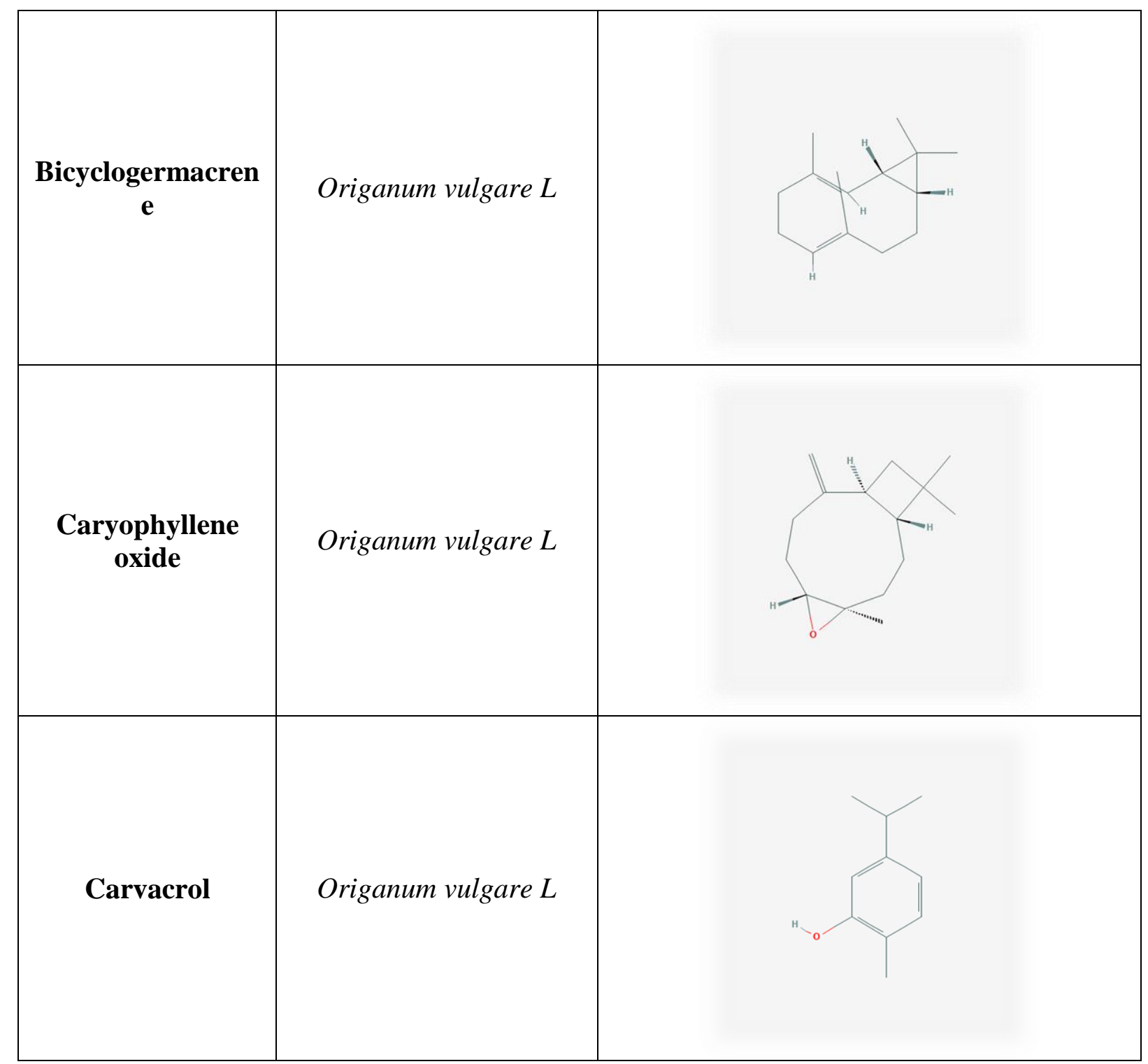

\section{Molecular Docking}

MVD automatically detects potential binding sites (cavities) using the cavity detection algorithm. To mechanize benchmarking, cavities within a 30x 30x30 3 cube centered at the experimentally known ligand position were used. The cavities found by the cavity detection algorithm are actively used by the search algorithm guided differential evolution to focus the search, to that specific area during the docking stimulation. For each ligand docking, the best orientation for the ligand-protein complex was analyzed and hydrogen bonds were identified and labeled. The ligand energy was inspected and analyzed using MVD score, a linear combination of E-inter (steric, Vander Waals, hydrogen bonding and electrostatic interactions) and E-intra (torsion, sp2 - sp2, hydrogen bonding, Vander Waals and electrostatic interaction). In this study, molecular docking MVD software 
(https://omictools.com) was used to investigate the molecular interaction between terpene compounds and protease enzyme. This software enables three-dimensional observation of the interaction between terpene and protease enzyme virus of the amino acids participating in the interaction between the functional and functional groups on the terpene molecules. In the present study, all docking conditions for terpene compounds, the number of interactions, the interaction area, the protease enzyme and the rate of docking were considered to minimize error.

\section{Steam therapy}

Steam Inhalation is a method in which a patient is treated by receiving aqueous incense of medicinal plants. This type of treatment is more important for the treatment of diseases related to the respiratory system and skin diseases, and due to the possibility of drug absorption from the capillary network of the nose, it is possible to treat many diseases with this method. Inhalation, use of incense through the respiratory tract can be considered as one of the methods of entering and absorbing active plant substances or chemical molecules into the body. Among the advantages that can be considered for this method are non-invasiveness, high speed of drug action, immediate absorption through arteries, nerves, mucosa and skin.

\section{Results}

\section{Docking Results}

All of the studied compounds have been linked to 7 important amino acids in the protected position of the enzyme flap. Among them, the binding energy of three amino acids is very high. These three amino acids are Asparagine151, Aspartate153 and Phenylalanine294. According to the results in the table 3, these compounds are connected to 18 residues, of which all compounds are connected to 7 residues, which we refer to the amount of binding energy. In Dithymoquinone, the amount of binding energy to Asn151 is -16, Asn156 is -11, Phe294 is -11 , Ser158 is -3.3 . In Farnesene, the amount of binding energy to Asn151 is -15 , Asn156 is -9.2, Phe294 is -10 , Ser158 is -2.6. In Spathulenol, the amount of binding energy to Asn151 is -19 , Asn156 is -10.5 , Phe294 is -13 , Ser158 is -5.3 . In Chamazulene, the amount of binding energy to Asn151 is -17.4, Asn156 is -11.6, Phe294 is -14.3, Ser158 is -3.9. In Enyn-dicycloether, the amount of binding energy to Asn151 is -13.7, Asn156 is -16, Phe294 is 6.1, Ser158 is -2.5. In Luteolin, the amount of binding energy to Asn151 is -20, Asn156 is - 
10, Phe294 is -6.6 , Ser158 is -2.3 . In Thymol, the amount of binding energy to Asn151 is -19, Asn156 is -14.3 , Phe294 is -6.9 , Ser158 is -6.1 . In Geranyl_acetate, the amount of binding energy to Asn151 is -15.8 , Asn156 is -10.3 , Phe294 is -16 , Ser158 is -4.5 . In Borneol acetate, the amount of binding energy to Asn151 is -19 , Asn156 is -14 , Phe294 is -12 , Ser158 is -3.6. In beta-Caryophyllene, the amount of binding energy to Asn151 is -14, Asn156 is -14, Phe294 is -13.7 , Ser158 is -3.3 . In beta-Bourbonene, the amount of binding energy to Asn151 is -19 , Asn156 is -10 , Phe294 is -14 , Ser158 is -1.9. In Germacren D, the amount of binding energy to Asn151 is -16 , Asn156 is -10 , Phe294 is -11.7 , Ser158 is -3.2. In Bicyclogermacrene, the amount of binding energy to Asn151 is -15 , Asn156 is -14.3 , Phe294 is -11.4, Ser158 is -3.8. In Caryophyllene oxide, the amount of binding energy to Asn151 is 18.7, Asn156 is -13.8 , Phe294 is -12 , Ser158 is -3.4. In Carvacrol, the amount of binding energy to Asn 151 is -11 , Asn156 is -7.6 , Phe294 is -19 , Ser158 is -0.8 . However, the amount of binding energy of each compound is different from that of amino acids, and the result of all ester and hydrogen bonds is the total binding energy, or moldock score, as shown in Table 2. The results of this study indicate the strong interactions of drugs in the enzymatic flap conserved region. Among the interactions that occur in these regions are often van der Waals, the binding of three important amino acids due to their presence in the highly conserved region has an active site and a key role in enzymatic catalysis. The results of these compounds are summarized in Tables 2,3 and 4.

Table 2. The sum of the moldockscores resulting from the interaction of compounds and protease enzyme

\begin{tabular}{lcccc}
\hline \multicolumn{1}{c}{ Compound Name } & Total Energy & Ester Bond & Hydrogrn Bond & Electrostatic Bond \\
\hline Dithymoquinone & -97 & -100 & -7 & 0 \\
Farnesene & -85 & -90 & 0 & 0 \\
Spathulenol & -82 & -90 & -2 & 0 \\
Chamazulene & -71 & -75 & 0 & 0 \\
En-yn-dicycloether & -75 & -69 & 0 & 0 \\
Luteolin & -82 & -90 & -5 & 0 \\
Thymol & -55 & -56 & -4 & 0 \\
Geranyl_acetate & -86 & -81 & -5 & 0 \\
Borneol acetate & -77 & -74 & -5 & 0 \\
beta-Caryophyllene & -72 & -72 & 0 & 0 \\
beta-Bourbonene & -78 & -80 & 0 & 0 \\
Germacren D & -70 & -75 & 0 & 0 \\
Bicyclogermacrene & -71 & -75 & 0 & 0 \\
\hline Caryophyllene oxide & -74 & -75 & -3 & 0 \\
Carvacrol & -62 & -60 & -3 & 0 \\
\hline
\end{tabular}


Table 3.The amount of binding energy of amino acids in protease with compounds

\begin{tabular}{|c|c|c|c|c|c|c|c|c|c|c|c|c|c|c|c|c|c|c|}
\hline AminoAsid & Arg & Asn & Asp & Asp & Cys & Gln & Gln & Ile & Ile & Ile & Lys & Phe & Phe & Ser & Thr & Thr & Val & Val \\
\hline Residue ID & 105 & 151 & 153 & 295 & 156 & 107 & 110 & 106 & 106 & 152 & 102 & 8 & 294 & 158 & 111 & 292 & 104 & 157 \\
\hline Dithymoquinone & -1.2 & -16 & $11 . \overline{6}$ & & & -7.4 & -7.6 & & -13.3 & $5 . \overline{2}$ & & -1.7 & $11 . \overline{5}$ & -3.3 & -0.8 & & -5.5 & \\
\hline Farnesene & -2.0 & -14.9 & -9.2 & -0.8 & & -2.8 & -5.2 & & -7.8 & $4 . \overline{-}$ & & -3.8 & 10.3 & -2.6 & -1.9 & -0.5 & -2.5 & \\
\hline Spathulenol & & -19.0 & 10.5 & -0.5 & -0.4 & & -4.5 & & -2.4 & $5 . \overline{-}$ & & -1.6 & $13 . \overline{-}$ & -5.3 & -2.0 & -0.7 & -2.4 & \\
\hline Chamazulene & & -17.4 & $11 . \overline{8}$ & & & & -4.6 & & -0.7 & $5 . \overline{4}$ & & -2.2 & $14 . \overline{3}$ & -3.9 & -0.3 & -0.6 & -1.4 & \\
\hline En-yn-dicycloether & -2.3 & -13.7 & 16.0 & & & & -0.5 & -4.8 & & $7 . \overline{2}$ & & -2.8 & -6.1 & -2.5 & & & -7.1 & \\
\hline Luteolin & -9.7 & -20.6 & $10 . \overline{-}$ & & -0.8 & -6.1 & -7.7 & -12.9 & & $6 . \overline{6}$ & -0.4 & -2.5 & -6.6 & -2.3 & -0.8 & & -6.2 & $0 . \overline{4}$ \\
\hline Thymol & & -19.0 & $14 . \overline{-}$ & & & & -0.4 & & -0.7 & $\begin{array}{r}- \\
5.7\end{array}$ & & -0.8 & -6.9 & -6.1 & -0.4 & & -1.3 & \\
\hline Geranyl_acetate & & -15.8 & 10.3 & -1.8 & & & -2.9 & & -1.0 & $6 . \overline{-}$ & & -3.3 & 16.3 & -4.5 & -1.7 & -5.0 & -1.1 & \\
\hline Borneol acetate & & -19.0 & $14 . \overline{-}$ & -0.3 & & & -0.7 & & -1.8 & $6 . \overline{-}$ & & -3.5 & $12 . \overline{-}$ & -3.6 & -1.7 & & -0.8 & \\
\hline beta-Caryophyllene & & -14.2 & $14 . \overline{-}$ & & & & -3.2 & & -0.9 & $6 . \overline{3}$ & & -1.8 & $13 . \overline{-}$ & -3.3 & -0.8 & & -1.5 & \\
\hline beta-Bourbonene & & -19.2 & $10 . \overline{-}$ & -0.4 & & & -4.9 & & -2.0 & $3 . \overline{-}$ & & -4.0 & $14 . \overline{2}$ & -1.9 & -1.2 & -0.3 & -0.5 & \\
\hline Germacren D & & -16.2 & $10 . \overline{-}$ & -0.4 & -0.4 & -0.3 & -5.4 & & -4.1 & $6 . \overline{2}$ & & -2.4 & $11 . \overline{-}$ & -3.2 & -2.0 & & -1.7 & \\
\hline Bicyclogermacrene & & -15.1 & $14 . \overline{3}$ & & & & -1.5 & & -0.9 & $6 . \overline{5}$ & & -1.9 & $11 . \overline{4}$ & -3.8 & -0.7 & & -2.0 & \\
\hline Caryophyllene oxide & & -18.7 & 13.8 & -0.4 & & & -1.8 & & -1.2 & $0 . \overline{-}$ & & -5.9 & 12.0 & -3.4 & -0.9 & & -2.1 & \\
\hline Carvacrol & & -11.2 & -7.6 & -3.8 & & & -1.1 & & & $4 . \overline{6}$ & & -4.7 & $18 . \overline{-}$ & -0.8 & -5.3 & -1.4 & & \\
\hline
\end{tabular}

Table4. The amount of energy of hydrogen bind in compounds with water

\begin{tabular}{|c|c|c|c|c|c|c|}
\hline Water & $\begin{array}{l}\text { HOH } \\
408\end{array}$ & $\begin{array}{l}\text { HOH } \\
417 \\
\end{array}$ & $\begin{array}{l}\text { HOH } \\
440\end{array}$ & $\begin{array}{l}\text { HOH } \\
456\end{array}$ & $\begin{array}{l}\text { HOH } \\
463\end{array}$ & $\begin{array}{l}\text { HOH } \\
479\end{array}$ \\
\hline ID & 7 & 16 & 39 & 55 & 62 & 78 \\
\hline Dithymoquinone & & -6.9 & -7.9 & & 2.0 & 0.9 \\
\hline Farnesene & -1.1 & -1.4 & 0.4 & & -2.6 & -0.6 \\
\hline Spathulenol & -0.5 & 2.9 & -1.8 & -0.4 & -5.1 & \\
\hline Chamazulene & & -0.4 & -1.6 & -0.9 & -1.9 & \\
\hline En-yn-dicycloether & & -1.2 & -1.3 & & -2.8 & -0.7 \\
\hline Luteolin & & & -1.7 & & -3.5 & -4.0 \\
\hline Thymol & & & -1.7 & & -1.7 & \\
\hline Geranyl_acetate & -0.7 & -0.5 & -5.6 & -0.8 & -1.0 & \\
\hline Borneol acetate & -0.8 & -1.2 & -4.1 & & -4.4 & \\
\hline beta-Caryophyllene & -2.0 & & -2.7 & & -1.4 & \\
\hline beta-Bourbonene & -0.7 & -1.9 & -2.2 & -0.3 & -1.8 & \\
\hline Germacren D & -0.7 & -2.7 & 4.5 & & -3.0 & \\
\hline Bicyclogermacrene & & -0.4 & -2.9 & & -2.1 & \\
\hline Caryophyllene oxide & -1.1 & & -3.1 & & & \\
\hline Carvacrol & -3.9 & -0.5 & 0.8 & -0.8 & -0.3 & \\
\hline
\end{tabular}


Table 5. The sum of the moldockscore resulting from the interaction of drugs and protease enzyme

\begin{tabular}{lcccc}
\hline \multicolumn{1}{c}{ Drug Name } & Total Energy & Ester Bond & Hydrogrn Bond & Electrostatic Bond \\
\hline Hydroxychloroquine & -87 & -86 & -7 & 0 \\
Favipiravir & -62 & -58 & -10 & 0 \\
\hline
\end{tabular}
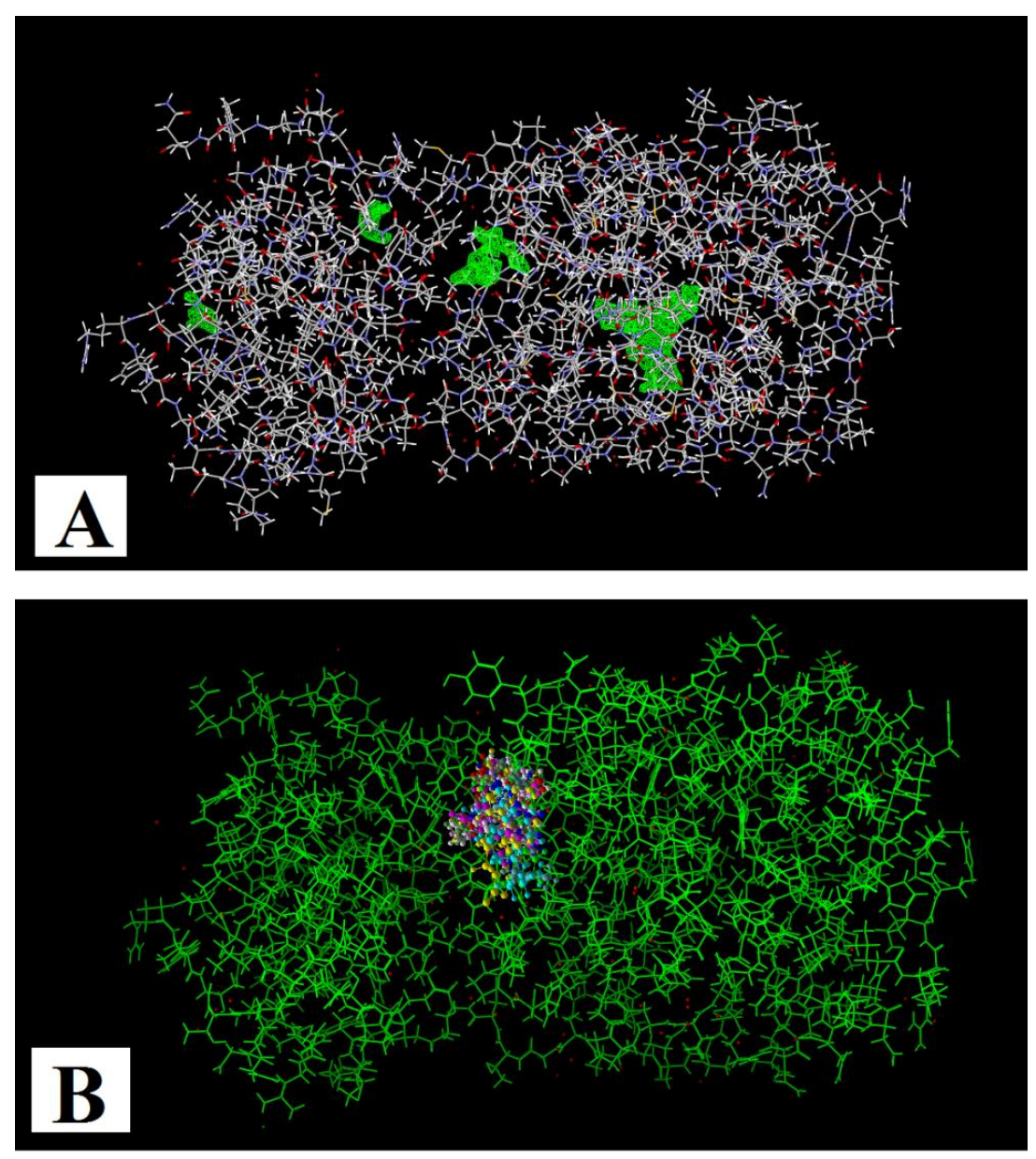

Figure 1.A:Detect 4 Cavity (is a protected area that is more interested in connecting to the Ligand) for

Connecting drugs B: Interactions between the compounds and COVID_19 protease are put into the middle cavity of the protease virus 


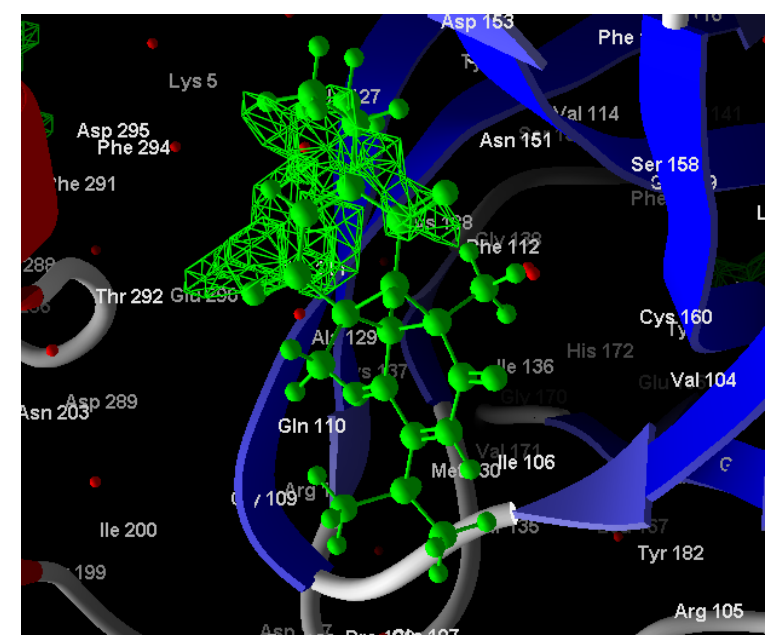

Figure2. Placement and binding of Nigella Sativa compound (dithymoquinone) to protease
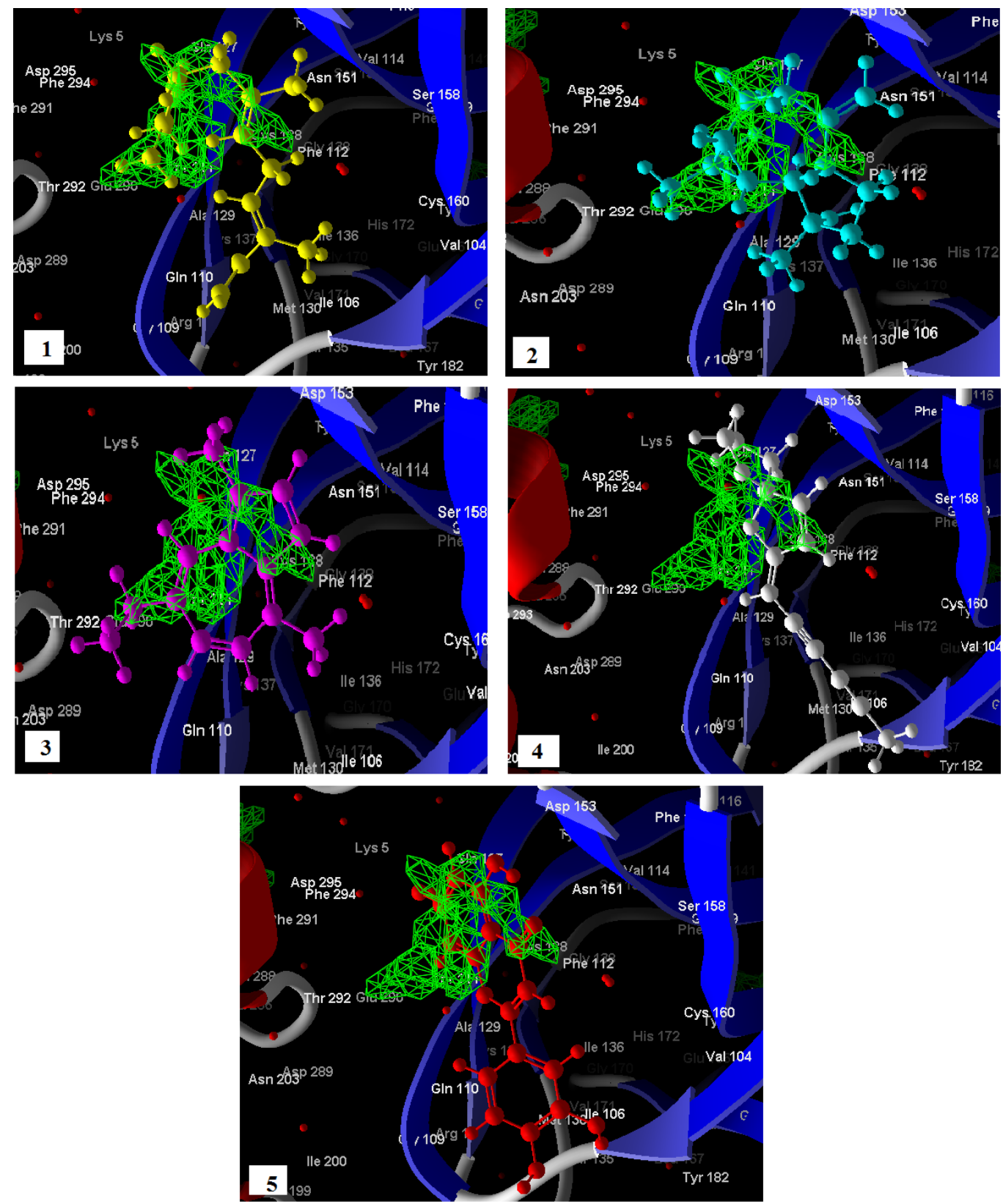
Figure 3. Placement and binding of Matricaria chamomilla compounds to protease: 1) Farnesene 2)Spathulenol 3) Chamazulene 4) En-yn-dicycloether 5) Luteolin
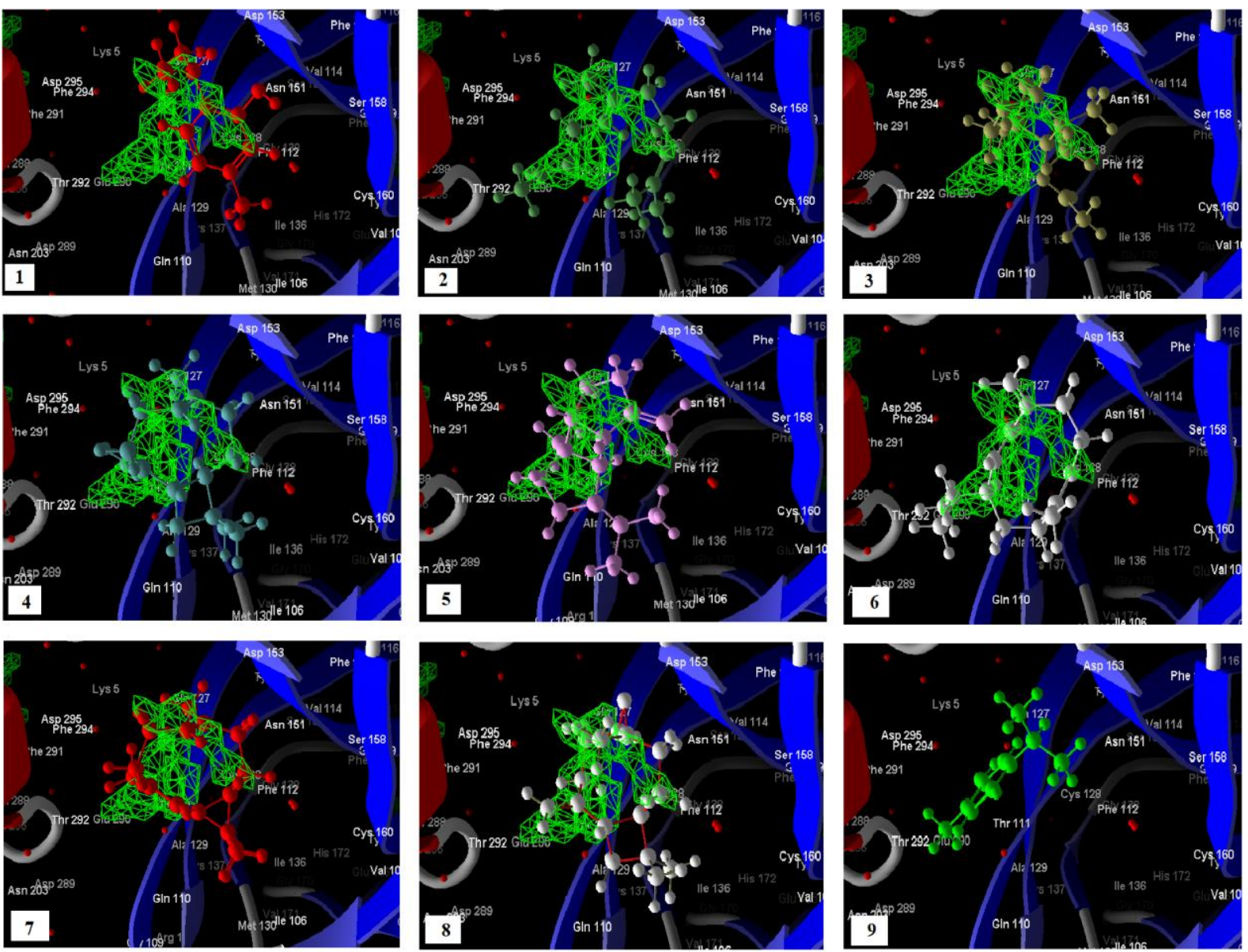

Figure 4. Placement and binding of Origanum vulgare L compounds to protease:1) Thymol 2) Geranyl_acetate 3) Borneol acetate 4) beta-Caryophyllene 5) beta-Bourbonene 6) Germacren D 7) Bicyclogermacrene 8) Caryophyllene oxide 9) Carvacrol

\section{Clinical Results}

In a clinical trial, a combination of the plants studied in this study received a specific percentage of 15 volunteers who were mildly or severely infected with the coronavirus and who had symptoms of pneumonia, Also severe cough and other symptoms of Covid_19. Some participants also took antibiotics and other medications to improve the symptoms of Covid_19. Finally, observations showed that in a few days after taking the drug, the symptoms of coronavirus disappeared in the body of patients receiving this compound and Their coughs went away. Also, in patients with hypoxia, their oxygen levels increased with 
the use of this compound. In this article, we have included three cases of CT scans of patients Figure $(5,6,7)$.

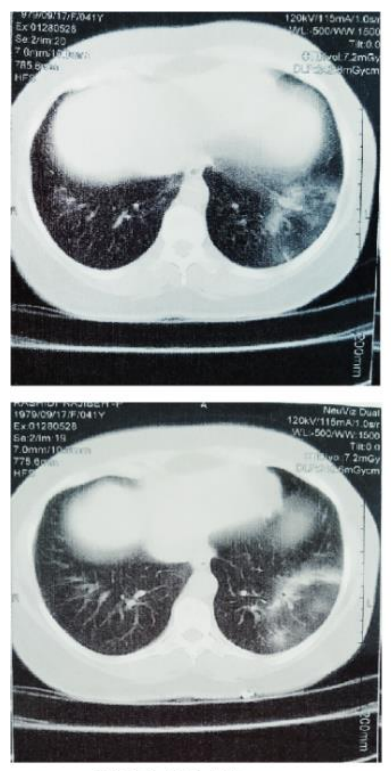

$2020 / 09 / 17$

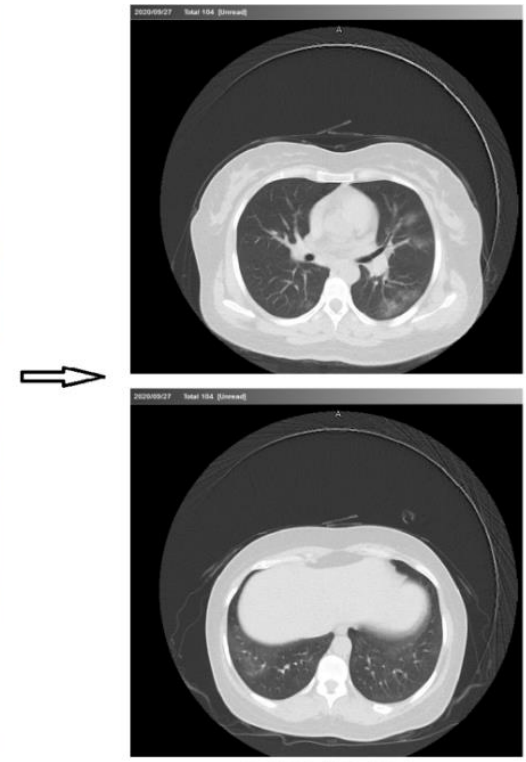

$2020 / 09 / 27$

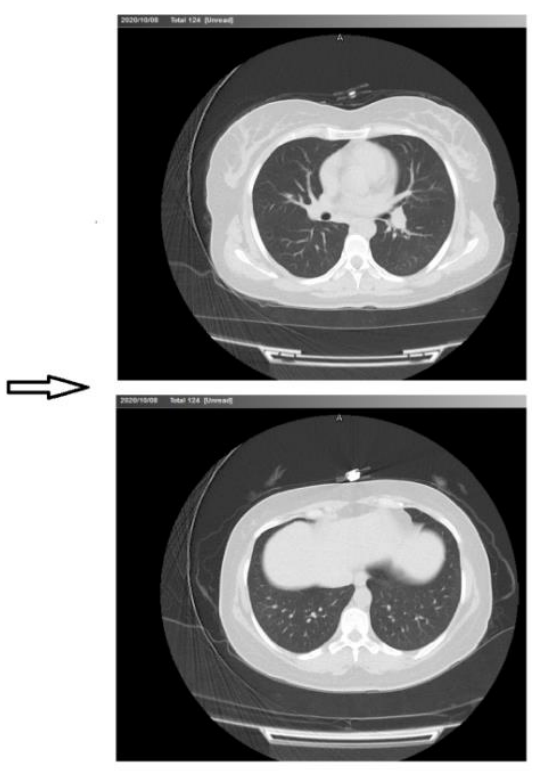

$2020 / 10 / 08$

Figure 5. CT scan of a 45-year-old patient with a lung infection who recovered after consuming the herbal composition
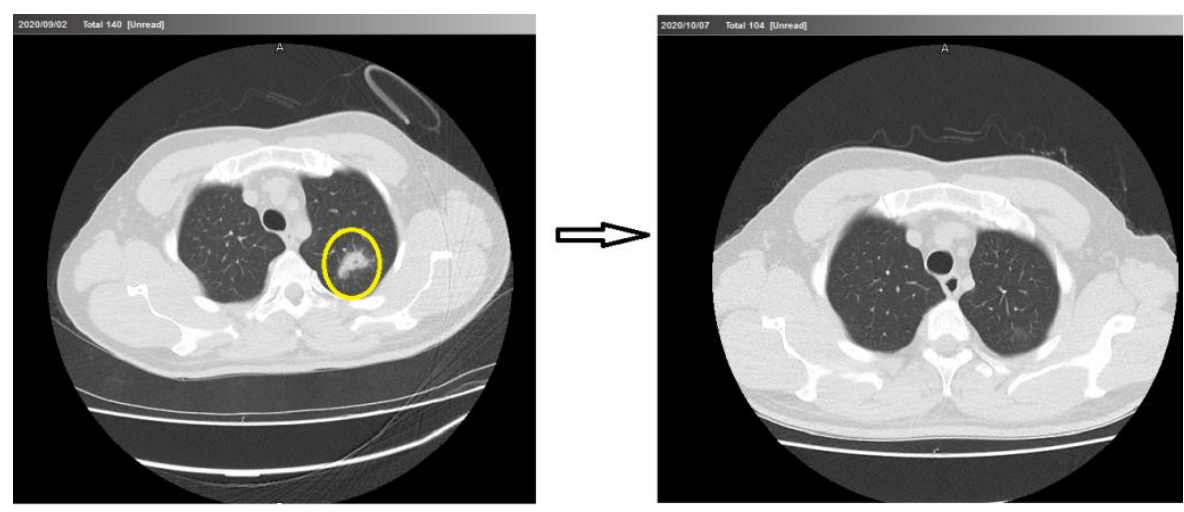

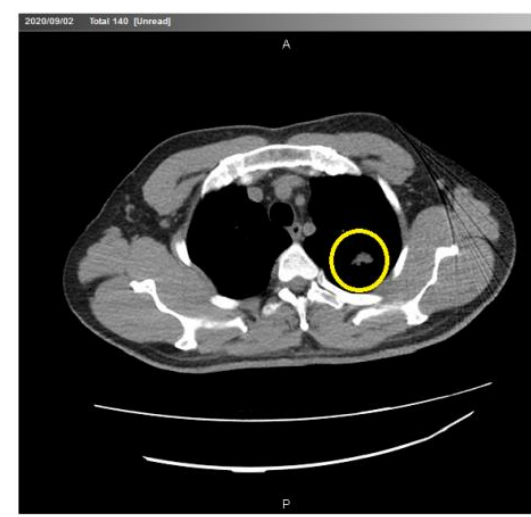

Before

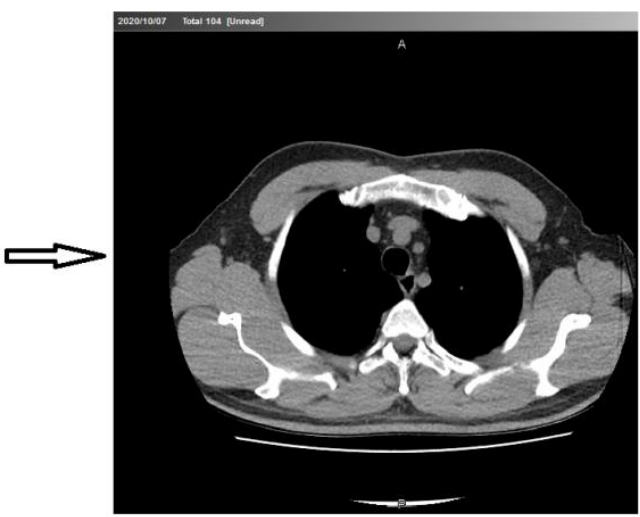

After 
Figure 6. CT scan of a 36-year-old patient with a lung infection who recovered after consuming the herbal composition
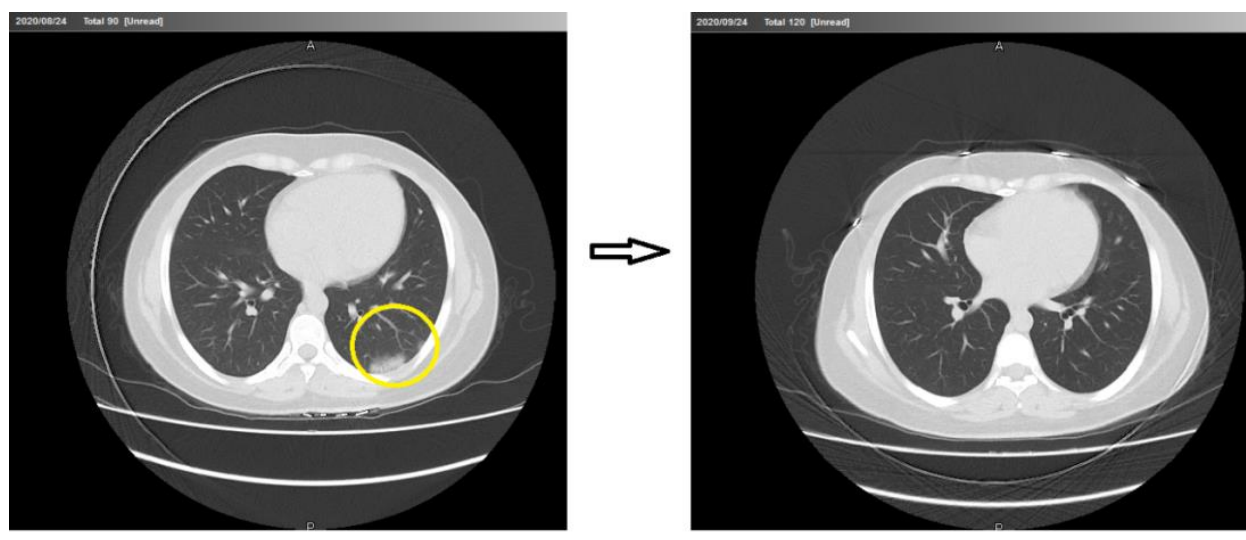

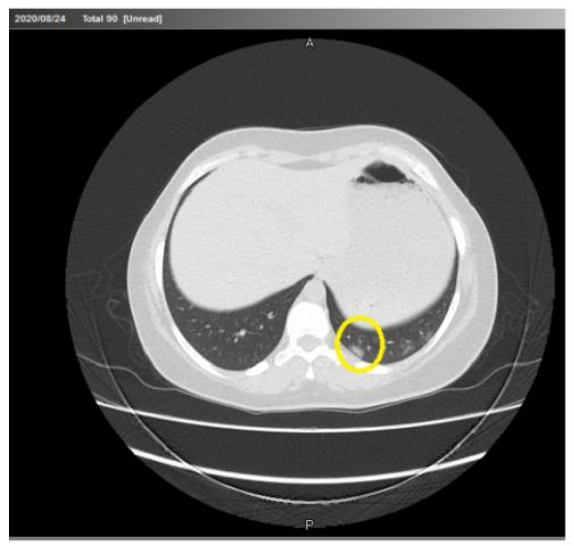

Before

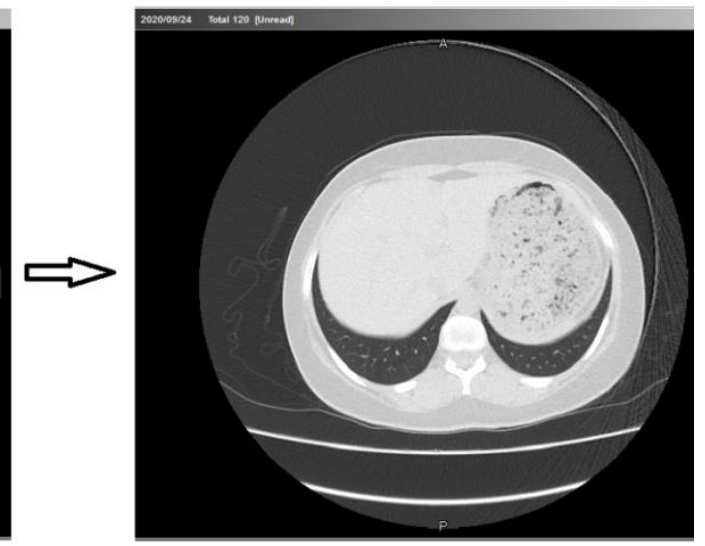

After

Figure 7. CT scan of a 27-year-old patient with a mild lung infection who recovered after consuming the herbal composition

\section{Discuss}

The use of herbal medicines in different countries is increasing day by day, and this is due to the proven effectiveness of these substances in human societies. Complementary therapy has been considered. Because corona affects the lungs, a drug that can enter the respiratory tract increases its inhibitory and therapeutic effects (Salem \& Hossain, 2000).Also, in this disease, pneumonia causes the severity of the disease. The aim of this study was to investigate the inhibitory effects of metabolites in the essential oils of three medicinal plants on corona virus protease. In this study, drugs were used that have previously been shown to have antiviral and anti-inflammatory effects and increase their immune system. Black seed plant with the scientific name of Nigella Sativa. Thymoquinone and thymol are important compounds in its 
essential oil. The most medicinal properties of this plant are related to thymoquinone (Malik et al, 1995). Anti-inflammatory, antioxidant, immune-boosting effects have led to the release of several pharmacological effects of black seed (B. Ali \& Blunden, 2003). Several studies have also confirmed its effects on boosting the immune system (Christou et al., 1989). In one study, oral administration and injection of its extract had anti-inflammatory and analgesic effects on laboratory mice (Hajhashemi et al, 2004). The use of black seed oil has antiviral effects on cytomegalovirus (Salem \& Hossain, 2000). Black seed oil has been reported to be effective against viruses by stimulating T cells (H. C. Su et al., 2001). Chamomile is one of the oldest medicinal plants known to man and dates back to ancient Greece. Chamomile has been introduced as a medicinal plant in all reputable pharmacopoeias and its medicinal properties have been studied. Chamomile has many properties, including digestive, healing, antiseptic, analgesic, anti-headache, antipyretic, anticonvulsant, anti-inflammatory (Alibabaei et al, 2014). The anti-inflammatory effects of chamomile alcohol extract have been investigated by inhibiting serum protein coagulation, and the results have shown that chamomile has strong anti-inflammatory effects. This plant has many terpenoid and flavonoid compounds, and the strong anti-inflammatory effects of these compounds have also been proven (Sadighara et al, 2013). Marjoram is one of the plants of the mint family, which is considered as one of the most important and best-selling medicinal and medicinal plants in the world. Recent findings suggest that the active ingredients of marjoram, in addition to having antibacterial, antifungal, antiviral, and anticancer properties, may also be effective in improving chronic rhinosinusitis, controlling diabetes, preventing inflammation, antuviral and cardiovascular disease. Pharmacological studies have shown that the high antioxidant potential of marjoram is due to the presence of phenolic monoterpenes such as thymol and carvacrol (Ocana-Fuentes et al, 2010; Raut \& Karuppayil, 2014). So far, none of the plants in this study have been clinically tested on coronavirus or in silico, But similar studies have been done with other synthetic drugs, some of which are mentioned here. Several antiviral medications: Zanamivir, Indinavir, Saquinavir, and Remdesivir show potential inhibitors and as a treatment for COVID-19 (Hall Jr \& Ji, 2020). Preliminary results from a study show that Indinavir and Remdesivir have the best docking scores, and a comparison of where the two drugs shows a near perfect dock in the overlap region of the protein pocket (Chang et al., 2020). In another research, the docking of ten plant molecules was studied, among them Oxyacanthine and Hypericin have shown good binding efficacy (Agrawal et al., 2020). In one study, Among the eight compounds studied, Lymecycline and Mizolastine were identified as possible inhibitors of coronavirus protease (Wafa \& Mohamed, 2020). In another study, 318 
phytochemicals performed by docking studies showed better inhibitory action on protease are Piperolactam, Quercetin ,glucoside ,Schaftoside ,Chrysoeriol, Isosakuranetin ,neohesperidoside, Delphinidin ,Petunidin ,Riboflavin, Oleanolic acid ,caffeoylquinic ,Absinthin ,Anabsinthin ,Dicaffeoylquinic acids (Joshi et al., 2020). Coronavirus proteases are attractive targets for the design of antiviral drugs. Coronavirus protease is activated as a dimer, and the binding of a inhibitor prevents it from becoming dimer, thus inhibiting this protein. The protein fold is similar to serine proteases like trypsin, but a cysteine amino acid and a nearby histidine perform the protein-cutting reaction and an extra domain stabilizes the dimer (Liu et al., 2020). The essential oils of these three plants can be introduced as drugs due to their effective ingredients and also their inhibitory effect on coronavirus protease. Due to the strong interaction of these natural compounds with enzymatic protected areas and special capabilities, these drugs can be considered as effective antiviral drugs that form their natural origin and will have the least side effects. They can be a good alternative or supplement to artificial and chemical drugs that have side effects.

\section{Conclusion}

According to the results of the present study, the compounds in the essential oils of the three medicinal plants can communicate with the important amino acids present in the protected area of the enzyme to prevent the activation of the new coronavirus protease. Also, due to their essential oil, they can enter the respiratory tract directly and have a double therapeutic effect.

\section{References}

Agrawal, A., Jain, N. K., Kumar, N., \& Kulkarni, G. T. (2020). MOLECULAR DOCKING STUDY TO IDENTIFY POTENTIAL INHIBITOR OF COVID-19 MAIN PROTEASE ENZYME: AN IN-SILICO APPROACH.

Ali, B., \& Blunden, G. (2003). Pharmacological and toxicological properties of Nigella sativa. Phytotherapy Research: An International Journal Devoted to Pharmacological and Toxicological Evaluation of Natural Product Derivatives, 17(4), 299-305.

Ali, I \& ,.Alharbi, O. M. (2020). COVID-19: Disease, management, treatment, and social impact. Science of The Total Environment, 138861 .

Alibabaei, Z., Rabiei, Z., Rahnama, S., Mokhtari, S., \& Rafieian-kopaei, M. (2014). Matricaria chamomilla extract demonstrates antioxidant properties against elevated rat brain oxidative status induced by amnestic dose of scopolamine. Biomedicine \& Aging Pathology, 4(4), 355360 . 
Chang, Y.-C., Tung, Y.-A., Lee, K.-H., Chen, T.-F., Hsiao, Y.-C., Chang, H.-C., . . Y Yu, J.-Y. (2020). Potential therapeutic agents for COVID-19 based on the analysis of protease and RNA polymerase docking.

Christou, N. V., Tellado-Rodriguez, J., Chartrand, L., Giannas, B., Kapadia, B., Meakins, J . . , , Gordon, J. (1989). Estimating mortality risk in preoperative patients using immunologic, nutritional, and acute-phase response variables. Annals of surgery, 210(1), 69 .

Hajhashemi, V., Ghannadi, A., \& Jafarabadi, H. (2004). Black cumin seed essential oil, as a potent analgesic and antiinflammatory drug. Phytotherapy Research: An International Journal Devoted to Pharmacological and Toxicological Evaluation of Natural Product Derivatives, 18(3), 195-199.

Hall Jr, D. C., \& Ji, H.-F. (2020). A search for medications to treat COVID-19 via in silico molecular docking models of the SARS-CoV-2 spike glycoprotein and 3CL protease. Travel medicine and infectious disease, 101646.

Benchmarking sets for molecular docking. Journal .(2006) .Huang, N., Shoichet, B. K., \& Irwin, J. J .of medicinal chemistry, 49(23), 6789-6801

Joshi, T., Sharma, P., Mathpal, S., Pundir, H., Bhatt, V., \& Chandra, S. (2020). In silico screening of natural compounds against COVID-19 by targeting Mpro and ACE2 using molecular docking. Eur. Rev. Med. Pharmacol. Sci, 24, 4529-4536.

Lengauer, T., \& Rarey, M. (1996). Methods for predicting molecular complexes involving proteins. Curr. Opin. Struct. Biol, 5, 402-406.

Liu, X., Zhang, B ,.Jin, Z., Yang, H., \& Rao, Z. (2020). The Crytal Structure of 2019-NCoV Main Protease in Complex with an Inhibitor N3. RCSB Protein Data Bank.

Malik, S., Hasan, S. S., Choudhary, M. I., Ni, C.-Z., \& Clardy, J. (1995). Nigellidine-a new indazole alkaloid from the seeds of Nigella sativa. Tetrahedron letters, 36(12), 1993-1996 .

Ocana-Fuentes, A., Arranz-Gutierrez, E., Senorans, F., \& Reglero, G. (2010). Supercritical fluid extraction of oregano (Origanum vulgare) essentials oils: anti-inflammatory properties based on cytokine response on THP-1 macrophages. Food and chemical toxicology, 48(6), 15681575 .

Raut, J. S., \& Karuppayil, S. M. (2014). A status review on the medicinal properties of essential oils. Industrial Crops and Products, 62, 250-264 .

Sadighara, P., Barin, A., JAHED, G., \& Farjadmand, F. (2013). Assessment of antioxidant capacity and anti-inflammatory of alcoholic extraction of chamomile, morus, marshmallow, borage and rosemary .

Salem, M. L., \& Hossain, M. S. (2000). Protective effect of black seed oil from Nigella sativa against murine cytomegalovirus infection. International journal of immunopharmacology, 22(9), 729-740.

Sexton, N. R., Smith, E. C., Blanc, H., Vignuzzi, M., Peersen, O. B., \& Denison, M. R. (2016). Homology-based identification of a mutation in the coronavirus RNA-dependent RNA polymerase that confers resistance to multiple mutagens. Journal of virology, 90(16), 74157428 .

Sharifi-Rad, J., Sureda, A., Tenore, G. C., Daglia, M., Sharifi-Rad, M., Valussi, M „.Ademiluyi, A. O. (2017). Biological activities of essential oils: From plant chemoecology to traditional healing systems. Molecules, 22(1), 70 .

Su, H. C., Nguyen, K. B., Salazar-Mather, T. P., Ruzek, M. C., Dalod, M. Y., \& Biron, C. A. (2001). NK cell functions restrain T cell responses during viral infections. European journal of immunology, 31(10), 3048-3055 .

Su, S., Wong, G., Shi, W., Liu, J., Lai, A. C., Zhou, J., Gao, G. F. (2016). Epidemiology, genetic recombination, and pathogenesis of coronaviruses. Trends in microbiology, 24(6), 490-502.

Wafa, T., \& Mohamed, K. (2020). Molecular Docking study of COVID-19 Main Protease with Clinically Approved Drugs. 